\title{
Introduction to Mathematics and Geosciences: Global and Local Perspectives, Volume I
}

\author{
Rafael Orive, ${ }^{1,2}$ María L. Osete, ${ }^{3,4}$ Jesús I. Díaz, ${ }^{5,6}$ and José Fernández ${ }^{3}$
}

Mathematics plays a fundamental role in all scientific fields and, of course, in the Geosciences. This has been especially well known since, for example, the beginning of geodesy in the Greek era (VANíčEK and KRAKIWSKY 1992) or in the pioneering studies of planet Earth's interior and the description of the Earth's potential fields (geomagnetic and gravimetric). We can remember that Johann Carl Friedrich Gauss (1777-1855), who is recognized as one of the history's most influential mathematicians, had a remarkable influence in many fields of mathematics and science. He contributed significantly to the development of modern geophysics. In the field of geomagnetism, he developed the first method to obtain absolute geomagnetic measurements, worked out the mathematical theory for separating the inner and outer sources of Earth's magnetic field, and founded the "Magnetischer Verein" (Magnetic Union). Another pioneering natural topic was exploring Earth's interior using seismic waves for analyzing the Earth's inner structure and discovering underground resources. For instance, in 1936, the

1 Instituto de Ciencias Matemáticas (ICMAT), CSIC-UAMUCM-UC3 M, C. Nicolás Cabrera 13-15, 28049 Madrid, Spain. Email: rafael.orive@icmat.es

2 Departamento de Matemáticas, Facultad de Ciencias, Universidad Autónoma de Madrid, 28049 Madrid, Spain.

3 Institute of Geosciences (IGEO) (CSIC, UCM), Plaza de Ciencias 1\&3, 28040 Madrid, Spain. E-mail: mlosete@fis.ucm.es; jft@mat.ucm.es

4 Departamento de Física de la Tierra I, Facultad de Ciencias Físicas, Universidad Complutense de Madrid, Plaza de las Ciencias 1, 28040 Madrid, Spain.

5 Instituto de Matemática Interdisciplinar, Facultad de Ciencias Matemáticas, Universidad Complutense de Madrid, Plaza de Ciencias 3, 28040 Madrid, Spain. E-mail: ji_diaz@mat.ucm.es

6 Departamento de Matemática Aplicada, Facultad de Ciencias Matemáticas, Universidad Complutense de Madrid, Plaza de las Ciencias 3, 28040 Madrid, Spain.
Danish mathematician Inge Lehman was the first to interpret $P$ wave arrivals, which inexplicably appeared in the $\mathrm{P}$ wave shadow of the Earth's core as reflections at an inner core, and discovered the Earth's solid inner core by studying these anomalies. Now it is natural to use geophysical methods and remote sensing in exploring our soils to find water, oil, minerals, etc., and to use advanced mathematical tools for interpreting observation results.

Therefore, as described by САмАсно et al. (2008a, b), mathematics is one of the branches of science, together with physics, chemistry, and Information Technology that studies and furthers knowledge of the Earth's structure and dynamics. This research is conducted in multidisciplinary studies that use and incorporate the most advanced methods of those sciences, in the framework of, or in close cooperation with, the different branches of Earth sciences such as geology, geophysics, and geodesy. The international dimension of this collaboration is evident (see, e.g., IUGS 2014; IUGG 2014).

This was the environment in which the Complutense International Seminar on "Earth Sciences and Mathematics" was organized and held in Madrid in 2006. The presentations at that meeting were published in two Pure and Applied Geophysics topical issues (САMACHO et al. 2008c, d).

Since 2006, Earth sciences and, therefore, their "mathematical needs" have evolved, marked to a very significant extent by the consolidated use of artificial satellites for the observation of Earth, which plays a more important role everyday (e.g., ASI 2014; CSA 2014; DLR 2014; ESA 2014a; JAXA 2014; NASA 2014). Satellite observation is evolving from regional-scale coverage at medium or low spatial resolution, to regional-scale and global-scale coverage at medium and high spatial resolution and local- 
scale coverage at ultra-high resolution. This evolution is bringing new needs of techniques/methodologies for data fusion (to obtain a unique, new data set by combining several sets from different satellites or from satellites and terrestrial observations) and for the joint interpretation of data of different sources (space and terrestrial) and kinds (displacements, gravity changes, seismicity,...).

Satellite synthetic aperture radar interferometry (InSAR) can serve as a typical example of this evolution, considering second-generation satellites (see, e.g., ASI 2014; DLR 2014; ESA 2014b; SANSOSTI et al. 2014). Data fusion is probably the only tool in this case to obtain a 3D displacement field with high spatial resolution (see, e.g., SAmsonov et al. 2008) or to obtain a continuous time series of displacement data for a long time period (see, e.g., Samsonov and D'Oreye 2012; SAmsonov et al. 2014). In addition, joint data interpretation is a powerful tool for studying Earth's active phenomena (see, e.g., САмасHO et al. 2011).

Advances in geomagnetic field knowledge in recent times have come with the combined use of satellite data about magnetism and the world geomagnetic observatories network (INTERMAGNET) developed for the present International Geomagnetic Reference Field: IGRF11 (FInLAY 2010). A spherical harmonic expansion is truncated at degree 13 (195 coefficients). The Swarm mission, launched in November 2013, is providing unprecedented insight into the complex workings of Earth's magnetic field (ESA 2014c, d). Advanced models based on Swarm data describing each of the various sources of the measured field are being developed, and will lead to new insight into many natural processes, from those occurring deep inside the planet, to weather in space caused by solar activity. In turn, this information will yield a better understanding of why the magnetic field is weakening.

At present, mathematical models and methods are required for diverse studies such as global change models, geodynamo models, flows in porous media, wave propagation in continua medium, riskmap analysis in hazards, time series analysis, dynamics of interfaces, studies with multiples scales, nonlinear analysis in the Earth sciences, fractal analysis in geological structures, stochastic models for processing data, etc.
Simultaneously, new problems (such as fracking), or the continental or global data sharing promoted by different initiatives such as CoPERnicus (2014), the Global Earth Observation System of Systems (GEOSS 2014), the European Plate Observing System (EPOS 2014), or SUPERSITES (2014), are posing new needs.

During the year 2013, at the initiative of American and Canadian research mathematical institutes and societies, 2013 was declared a thematic year in Mathematics and the Planet Earth (MPE2013 2014), under the patronage of UNESCO. More than 120 mathematical organizations around the world took part in MPE2013 and gave the public and schools an exciting opportunity to discover and experience many aspects of mathematics, including its applications on life and Earth sciences, its relevance on societal and environmental issues, the impact of mathematics research on thematic topics specific to climate change, remote sensing, mathematical modelling to eradicate diseases as well as the global trends, and perspectives on mathematics for sustainable development.

The four MPE2013 subthemes were a planet to discover; a planet supporting life; a planet organized by humans; and a planet in danger. Thus, the mathematical family found natural topics where mathematics play a fundamental role in describing different events in Earth sciences, in particular, in the first and the last theme.

To mark the MPE2013, the Institute of Mathematical Sciences (ICMAT) and Institute of Geosciences (IGEO), under the patronage of the Spanish Council for Scientific Research (CSIC), Madrid Autonomous University (UAM), the Institute of Interdisciplinary Mathematics (IMI), Madrid Complutense University (UCM), and Technical University of Madrid (UPM) sought to highlight the aforementioned different aspects, the two-way street between Mathematics and Earth Sciences, by organizing the workshop "Mathematics and Geosciences: Global and Local Perspectives" in Madrid at ICMAT from 4 to 8 November 2013. The Organizing Committee's main idea, when it proposed such a title, was to emphase the combination of two different types of approaches: the global approaches that study natural phenomena arising from the whole planet (such as the 
climate, geomagnetism, and so on) and the almost infinite collection of local aspects, which are associated with natural, ecological, and even economic studies on more concrete problems. The following topics were addressed: climatology and paleoclimatology, oceanography, geomagnetic field, the Earth's rotation, remote sensing, natural hazards, structure and geodynamics, renewable energies, and social and environmental aspects. These topics were addressed from a global perspective on the Earth as well as from a local point of view of the Geosciences. Scientists from both areas, Mathematics and Earth Sciences, participated in this activity seeking to gain and learn from their work and proposing partnerships.

This is the first volume of the Topical Issue on "Mathematics and Geosciences: Global and Local Perspectives" and contains 13 papers, most of which were presented at the Congress. They address different topics such as the percolation theory to observe microseismicity in fracking, fractional differential analysis to model complex dynamics, climate change, earthquake statistics, earth rotation, geomagnetic field, fire models, Mars exploration, numerical methods in meteorology, environmental conservation, and probabilistic models in the study of soils and fluid mechanics.

The first paper is about hydraulic fracturing (fracking); technical processes used to extract oil and gas from tightly sealed shale reservoirs using high pressures and a low viscosity fluid. Norris et al., model the injection of fluid in the fracking process using invasion percolation, which is a $2 \mathrm{D}$ square lattice of random bonds to model the sealed natural fractures. This model exhibits burst dynamics (small earthquakes) associated with the microseismic activity generated by fracking injections and shows the role of anisotropic stress distributions.

Tejedor et al., apply a new probability density function, the negative binomial distribution, to study the recurrence of large earthquakes. The new distribution has been applied to the study of the Parkfield seismic series, finding that it performs very similarly to other distributions. In contrast to other statistical models, the new distribution has a plausible physical meaning, that is, it is based upon the idea of the elastic-rebound and Markov modelling.

The paper by Velasco et al., deals with an interesting topic about Mars exploration. The authors present a new method of tomography-based signal analysis for the detection of events in the Martian atmosphere boundary layer, such as dust devils. The mathematical aspects are considered through fractional differential equations associated with diffusion processes and nonlocal problems. This area of work can provide important results of remarkable applications in the future.

In the paper by Fowler, the future evolution of atmospheric $\mathrm{CO}_{2}$ concentration is assessed on a millennial timescale by using a simple box model of the ocean carbon cycle. The model is based on two equations, one for the atmospheric $\mathrm{CO}_{2}$ concentration, and the other is for the carbonate concentration in the ocean. The model predicts a positive feedback in response to the long-term increase in atmospheric $\mathrm{CO}_{2}$ as a result of the decrease in the carbonate concentration and the buffering effect. A further consequence of the repartitioning of ocean carbon is a dramatic rise in atmospheric carbon dioxide on a millennial time scale.

Ferrándiz et al., present a very interesting review of the crucial problem of the natural sciences, that is, to improve the theory of the Earth's rotation in order to reach the accuracy requirements that must be fulfilled in the near future. The accurate determination and prediction of the Earth's orientation and motion in Space has been needed in a broad variety of fields ever since the advent of the Space Age. Applications of geodetic measurements to the determination of sea level variations, mass movements in oceans, ice sheets, terrestrial water storages, displacement fields associated with earthquakes, etc., are demanding far higher levels of accuracy. The authors broach the problem and suggest new research directions aimed at creating adequate use of the Earth's rotation theory.

The paper by De Santis and Qamili is an overview of the complex characteristics of the Earth's magnetic field investigated from a holistic approach. Within the framework of geosystemics and by using information-theoretic tools such as Shannon entropy or other nonlinear tools borrowed from critical phenomena, they explore the possibility of an imminent change of the geomagnetic field dynamical regime.

The paper by Campuzano et al., analyzes the main error sources in the geomagnetic dipole moment computation from palaeomagnetic data (the influence 
of the nondipole terms in the average approach, the inhomogeneous distribution of the current palaeomagnetic database, and the averaging procedure used to obtain the evolution of the dipole moment), using synthetic data from a global model based on instrumental and satellite data, the 11th generation of the International Geomagnetic Reference Field.

Oliver et al., introduce a new numerical method to solve mathematical models for wind field forecasting, coupling the predictions of the HARMONIE mesoscale model as the input data for an adaptive finite element in a local mass-consistent wind model specifically suited for complex terrains. These wind models serve as tools for studying several atmosphererelated problems, such as the effect of wind on structures, pollutant, fire spreading, and wind farm location. At present, the use of wind power to produce electric power involves using these mathematical tools to design the wind farms. The final goal of the paper is to validate the model in several realistic applications on Gran Canaria Island, Spain, with some experimental data obtained by the AEMET (Spanish Meteorological Agency) at its meteorological stations.

The paper by Ferragut et al., presents a global physical model (heat and mass transfer) available for analysis and numerical simulation of forest fire propagation. They incorporate data assimilation techniques to the numerical solution in near real time, which improve the simulation results. From a numerical and computational point of view, a highly interesting issue is the successful implementation of Yosida regularizations to approximate the enthalpy, which consists of a nonlinear multivalued maximal monotone operator.

Martín et al., consider fragmentation schemes inspired in the theoretical results and conjectures of Kolmogorov, which are applied to produce particle size distributions of a different nature depending on fragmentation parameters. A 2-D computer simulation packing method is applied to the resulting distributions and the void fraction is evaluated. They study the relation between the void fraction and the characteristic parameters of the fragmentation process.

In the paper by Arregui and Vázquez, the authors propose new efficient numerical methods to solve some mathematical models related to the opportunity of starting an industrial project that provides some uncertain benefits but also involves some irreversible environmental effects. Of course, both the environmental and the industrial project's benefits, are uncertain and governed by stochastic processes. The authors consider a finite element discretization of the equivalent PDE problem posed on a suitable bounded domain and, to add instantaneous effects, propose a Lagrangian active set method (ALAS). Finally, the numerical method is validated through qualitative properties theoretically proven in the literature for different examples.

Muñoz-Ortega et al., show the utility of performing different geometric measures of soil pore space using 3-D images in order to characterize the soil structure. They characterize in quantitative terms several geometric factors of great interest in the study of biological and physical processes, such as porosity, pore surface area, connectivity of the pores, and pore size distribution. For the latter objective, they use mathematical morphology tools.

The paper by Berselli et al., studies the motion of a quasi-incompressible fluid with several dispersed particles. This model plays a fundamental role in the dynamics of both oceanic and atmospheric flows. They consider a reduced multiphase model with particular attention to the right evaluation of physical parameters that make the approximation effective. The resulting model is used in direct numerical simulations and also large eddy simulations of a dambreak (lock-exchange) problem, which is a wellknown academic test case.

A second issue will be published later in 2015 with other reviewed papers presented at the workshop "Mathematics and Geosciences: Global and Local Perspectives".

We appreciate the great and generous work carried out by the many referees. They have worked in most of the cases, in the difficult intersection of two different fields such as Earth Sciences and Mathematics. The reviewers have been: G. Alguacil, J. Almendros, O. Arzel, L. D'Auria, S. Alvarez, G. Balasis, Y. Barkin, M. Bialecki, R. Carbonell, A. Castro, M. Crucifix, G. Diaz, J.I. Díaz, L. Dinis, J. Durany, P. García, R. Granero-Belinchón, R. Gross, A. Jimenez, J. von de Koppel, M. Korte, V. Kossobokov, M. Kravchenko, S. Lennartz-Sassinek, F. Luzón, J. Mandel, M. Montoya, J.J. Nieto, J.H. Ortega, J.F. Padial, A. Pazoto, E. Perfect, M. Sahimi, T. Sengupta, C. Trenchea, D.L. Turcotte, F. Valero, and C. Wang. 


\section{Acknowledgments}

We would like to take this opportunity to thank the Instituto de Ciencias Matemáticas and the Institute of Geosciences for their financial and technical support in organizing the Mathematical and Geosciences Congress, and especially E. Fuentes, E. Frechilla, and A. Chacón from ICMAT. We are also grateful to CEI Campus Moncloa (UCM-UPM) and CEI UAM + CSIC for their support. This workshop has been also partially supported with funds from research projects AYA2010-17448, MTM2011-26696, SEV-2011-0087, CGL2011-24790 of MINECO, MTM2011-26119 of the DGISPI (Spain), the UCM Research Group MOMAT (Ref. 910480), and the ITN FIRST of the Seventh Framework Program of the European Community's (grant agreement number 238702). The editors also thank Dr. Renata Dmowska and Ganesh Priyanka for the help, suggestions, and support received during the editing of this topical issue. Finally, we wish to thank all the authors of this volume for their contributions.

\section{REFERENCES}

ASI, Italian Space Agency (2014). http://www.asi.it/en.

CAmacho, A.G., Díez, J.I., Fernández, J. (2008a). Introduction: Linking Earth Sciences and Mathematics. Pure and Applied Geophysics, 165, no. 6, 997-1001. doi:10.1007/s00024-0080343-7.

Camacho, A.G., Díez, J.I., Fernández, J. (2008b). Introduction to Earth Sciences and Mathematics, Volume II. Pure and Applied Geophysics, 165, no. 8, 1459-1463. doi:10.1007/s00024-0080396-7.

Camacho, A.G., Díez, J.I., Fernández, J. (Editors) (2008c). Pure and Applied Geophysics Topical Issue "Earth Sciences and Mathematics. Volume I." 165, no. 6.

Camacho, A.G., Dítz, J.I., Fernández, J. (Editors) (2008d). Pure and Applied Geophysics Topical Issue "Earth Sciences and Mathematics. Volume II." 165, no. 8, 1459-1706.

Camacho, A. G., P. J. González, J. Fernández, and G. Berrino (2011). Simultaneous inversion of surface deformation and gravity changes by means of extended bodies with a free geometry: Application to deforming calderas, J. Geophys. Res., 116, B10401, doi:10.1029/2010JB008165.

COPERNICUS (2014). http://www.copernicus.en/main/overview/.

CSA, Canadian Space Agency (2014). http://www.asc-csa.gc.ca/ eng/.
DlR, National Aeronautics and Space Research Centre of the Federal Republic of Germany (2014). http://www.dlr.de/dlr/en/ desktopdefault.aspx/tabid-10376.

ESA, European Space Agency-Observing the Earth (2014a). http://www.esa.int/Our_Activities/Observing_the_Earth/.

ESA, European Space Agency, Sentinel-1 (2014b). http://www. esa.int/Our_Activities/Observing_the_Earth/Copernicus/ Sentinel-1.

ESA, European Space Agency-Observing the Earth (2014c). http://www.esa.int/Our_Activities/Observing_the_Earth/Swarm/ Swarm_reveals_Earth_s_changing_magnetism.

ESA, European Space Agency (2014d). ESA, 3rd Swarm Scientific meeting, June 2014, Denmark. http://congrexprojects.com/2014events/Swarm/home.

EPOS, European Plate Observing System (2014). http://www. epos-eu.org/.

Finlay, C. C. (2010). International Geomagnetic Reference Field: the eleventh generation. Geophys. J. Int. 183, 1216-1230.

GeOSS, Global Earth Observation System of Systems (2014). http://www.epa.gov/geoss/.

IUGG, International Union of Geodesy and Geophysics (2014). http://www.iugg.org/.

IUGS, International Union of Geological Sciences (2014). http:// www.iugs.org/.

JAXA, JaPAn Aerospace Exploration Agency (2014). http://global. jaxa.jp/.

MPE2013, Mathematics of Planet Earth (2014). http://mpe2013. org/.

NASA, National Aeronautics and Space Administration (2014). http://www.nasa.gov.

SAmsonov, S., and N. D’OreYe (2012), Multidimensional time series analysis of ground deformation from multiple InSAR data sets applied to Virunga Volcanic Province, Geophys. J. Int., 191(3), 1095-1108, doi:10.1111/j.1365-246X.2012.05669.x.

Samsonov, S.V., Tiampo, K.F.; Rundle, J.B. (2008). Application of DInSAR-GPS optimization for derivation of three-dimensional surface motion of the southern California region along the San Andreas fault. Computers and Geosciences, 34, 503-514, doi:10. 1016/j.cageo.2007.05.013.

Samsonov, S.V., Tiampo, K.F., Camacho, A.G., Fernández, J., GonZÁLEZ, P.J. (2014). Spatiotemporal analysis and interpretation of 1993-2013 ground deformation at Campi Flegrei, Italy, observed by advanced DInSAR. Geophysical Research Letters, 41, pp. 6101-6108, doi:10.1002/2014GL060595.

Sansosti, E., Berardino, P., Bonano, M., Calò, F., Castaldo, R., Casu, F., Manunta, M. Manzo, M., Pepe, A., Pepe, S., Solaro, G., Tizzani, P., Zeni, G., Lanari, R. (2014). How second generation SAR systems are impacting the analysis of ground deformation. International Journal of Applied Earth Observation and Geoinformation, 28, pp. 1-11, doi:10.1016/j.jag.2013.10. 007.

SUPERSITES (2014). http://supersites.earthobservations.org/.

VANíčeK, P. and Krakiwsky, E. (1992). Geodesy, the concepts. Second Edition. Elsevier, Amsterdam, The Netherlands, ISBN: 0-444-87777-0, 697 pp. 